\title{
Los procesos decisorios seguidos para el establecimiento de una agenda pública en Colombia
}

\section{The Decisive Processes Followed for the Establishment of a Public Agenda in Colombia}

Corina Duque Ayala *

Fecha de recepción: 14 de agosto de 2008

Fecha de aprobación: 18 de septiembre de 2008

\section{Resumen}

El presente artículo pretende dar a conocer, de manera general, los diferentes modelos y enfoques que influyen en la elaboración de las políticas públicas, y visualizar con algunos ejemplos colombianos, la manera como algunos procesos decisorios de las políticas públicas se han implementado en el país.

\section{Palabras clave}

Políticas públicas, agenda pública, modelos de desarrollo, seguridad democrática.

\section{Abstract}

This article aims to raise awareness, generally speaking, the different models and approaches that influence the shaping of public policies, and view with some Colombians examples, how some decision-making processes of public policies have been implemented in the country.

\section{Key words}

Public policy, public agenda, models of development, democratic security. 
A continuación se presenta una síntesis de las diferentes teorías, enfoques y modelos para la elaboración de las políticas públicas, para lo cual, el ensayo se desarrolla en tres partes: en la primera parte, gracias a un ejemplo práctico, "la política de la seguridad democrática", se visualizan las diferencias teóricas que existen entre el enfoque incrementalista o descriptivo y el racionalista $o$ prescriptivo, y se logra describir la forma como el actual gobierno ha venido tomando decisiones públicas, que son el resultado de incrementos marginales de los actores principales en los ámbitos político, social y económico, pero también producto de situaciones críticas coyunturales que gestaron estas decisiones, lo que hace que quede desvirtuada la teoría de que los cambios radicales en nuestro país, únicamente, se han logrado como consecuencia de la capacidad de raciocinio de los seres humanos. En otras palabras, las decisiones de política pública gubernamental en torno a la imperiosa necesidad de resolver el conflicto armado han sido más el fruto de una negociación política, que de un modelo económico previamente concebido por los académicos y los economistas.

En la segunda parte se desarrolla el tema de la forma como se establece una agenda pública, para lo cual nuevamente se utiliza un ejemplo práctico en Colombia y se analiza a la luz del enfoque descrito por Zahariadis, la teoría del "cubo de basura", para llegar a la conclusión de que efectivamente en nuestro país, tanto en las organizaciones públicas como en la gestión de los bienes públicos, se sigue una racionalidad que tiene en cuenta, por un lado, los problemas y, por otro, las soluciones y los actores en su conjunto, quienes son los que realmente han venido impulsando dichas soluciones. En algunos momentos coyunturales, estos actores observan cómo se les abre una "ventana de oportunidades" que les permiten hacer realidad sus sueños, plasmar sus ideas en leyes y decretos, lo que hace efectivas las soluciones propuestas, tal como ocurrió con la Asamblea Constituyente en 1991. Por último, se analiza el papel de algunos actores en la adopción de las decisiones, verificando quiénes han sido los protagonistas de los cambios y cuáles serán los nuevos paradigmas en la construcción de las políticas públicas.

Del análisis de estos tres aspectos: modelos para la toma de decisiones, enfoques para el establecimiento de políticas públicas y papel de los diferentes actores se concluye que para la implementación de las políticas públicas se deben tener en cuenta las creencias y los valores; además se deben entender las relaciones de las diferentes corrientes analizadas individualmente y en su actuar colectivo, para lograr grandes periodos de estabilidad, con esquemas realmente pluralistas y ampliamente democráticos, así como la crisis de los primeros modelos de elaboración de políticas públicas y la aparición de la estrategia de exploración combinada.

El enfoque de elaboración de las políticas públicas tiene aplicabilidad en la realidad colombiana. Para entenderlo se debe empezar por sintetizar las dos primeras teorías que aparecieron para el análisis de las políticas públicas, los modelos racionalista e incrementalista:

- El racionalista o prescriptivo se basa en el análisis de sistemas con fuerte inclinación a modelos cuantitativos; las ciencias administrativas y sus aplicaciones al derecho y a la administración pública, y la ciencia política con su orientación hacia el estudio de las instituciones. Los supuestos son: los métodos de la ciencia, el empirismo y el racionalismo son los que harán más racionales y efectivas las decisiones públicas.

- El incrementalista o descriptivo, que otorga mayor énfasis a la idea de las que las decisiones políticas son el resultado de incrementos marginales de los actores sociales.

Asimismo, se ha venido desarrollando un tercer enfoque que pretende superar los dos anteriores, al explicar los cambios radicales en determinados 
momentos históricos; este enfoque hoy en día se conoce como el enfoque multidisciplinario de Zahariadis, con tres elementos: la decisión racional, el marco de coaliciones de apoyo y las corrientes múltiples.

Al estudiar estos modelos en la realidad colombiana, se puede concluir que nuestros gobernantes al momento de diseñar las políticas públicas, utilizan de manera alterna uno y otro modelo, por cuanto no son excluyentes $y$, por el contrario, permiten realizar combinaciones y generar alternativas que resultan muy interesantes para proponer soluciones a problemas estructurales, tales como el del conflicto armado en Colombia.

Tal y como lo señala Etzioni:

[...] aunque los incrementalistas sostengan que la toma de decisiones implica una elección entre dos clases de modelos de decisión, debe señalarse que:

a) La mayoría de decisiones incrementales especifican o anticipan decisiones de carácter fundamental y,

b) que el valor acumulativo de las decisiones incrementales es influido en gran medida por su vinculación con las decisiones fundamentales (1967, pp. 265-283).

Si se investigaran los cambios incrementales en una sociedad como la colombiana, se terminaría por concluir que este cúmulo de cambios, además de ser el resultado de incrementos marginales de Ios actores principales, puede llegar a terminar siendo la ejecución de las tendencias iniciadas en momentos sociales críticos, tales como la Constituyente de 1991, cuando se debieron tomar decisiones fundamentales que permitieron consolidar los resultados esperados de apertura democrática, en un tiempo estimado por nuestros científicos con enfoque racionalista.

En Colombia, un ejemplo una decisión fundamental es la adopción de la política de la Seguridad Democrática, que consiste en incrementar y pro- fesionalizar las Fuerzas Armadas de Colombia, para realizar una confrontación efectiva a los actores armados al margen de la Ley. Esta decisión se tomó iniciando el gobierno Uribe, para lo cual se tuvo que lograr la adición del presupuesto para la defensa nacional, por parte de los congresistas. Si se observan los incrementos del presupuesto de defensa en estos cinco años de gobierno del presidente Álvaro Uribe Vélez, el primer incremento correspondiente al año 2002 fue realmente significativo y a partir de los años siguientes, las variaciones porcentuales con respecto al PIB se han mantenido constantes año tras año.

La decisión fundamental de hacer frente a los actores armados, vía confrontación armada, fue tomada en 2002 y las decisiones incrementales sucesivas relacionadas con esta materia, tales como "el proceso de paz con las autodefensas", "la atención integral a las familias desplazadas", "el programa para reinsertados", en últimas, han ratificado dicha decisión fundamental de lograr la "seguridad democrática"; aunque parecieran ser un conjunto de decisiones incrementales, sin mucha conexión, en efecto, son la ejecución de una decisión fundamental previa, que se refiere a lograr el cese del conflicto armado, enfrentándolos con "garrote y zanahoria".

Cuando en Colombia se decidió adoptar una política de seguridad democrática se le dio un papel más activo a la sociedad colombiana y así luchar junto con el Estado contra los insurgentes y demás grupos armados ilegales. De igual forma, se planteó la necesidad de fortalecer la presencia de las Fuerzas Militares a lo largo del territorio nacional y la colaboración de la ciudadanía en relación con esquemas de seguridad e inteligencia militar para obtener el éxito en la confrontación bélica y concomitante a este esquema de confrontación, la desmovilización de los grupos armados al margen de la Ley. Por esta razón, se incluyó el programa de red de cooperantes y el ofrecimiento de recompensas a los informantes; asimismo, se estimularon 
los beneficios para los reinsertados, se crearon las unidades de soldados profesionales y campesinos, todo gracias al aumento del presupuesto a la defensa nacional y a los recursos provenientes del Plan Colombia, adoptado por el gobierno de Estados Unidos.

En el segundo año de gobierno, se tomaron decisiones incrementales, tales como la elaboración de un Plan de Atención Integral a las familias desplazadas y un plan para lograr la entrega de las autodefensas, a fin de iniciar un proceso de juzgamiento y reparación a las víctimas. Algunos asesores del gobierno, entre ellos el actual Consejero de Paz, Luis Carlos Restrepo, reconocido académico y científico social de la escuela del racionalismo, presentó al gobierno una investigación exhaustiva de las causas del conflicto armado en Colombia, en la que se recopiló información desde los años cincuenta, con la muerte del caudillo Jorge Eliécer Gaitán; también se realizó un mapeo del conflicto, en el que indicaban las acciones subversivas ocurridas veinte años atrás. El resultado fue una infinidad de hechos, causas y consecuencias, que en su momento, sobrepasaron cualquier acción coyuntural del gobierno de turno, razón por la cual se decidieron acciones incrementalistas y se escogieron aquellas regiones donde el conflicto se había recrudecido en los últimos dos años; sin embargo, esto trajo como consecuencia que se ignoraran aquellas regiones donde los actores armados ilegales habían hecho presencia esporádica.

Es importante recordar que un incrementalista "puro", cuando decide evaluar sus decisiones para verificar si fueron efectivas las medidas para aliviar los problemas sociales coyunturales, termina utilizando las herramientas de control y evaluación diseñadas por los racionalistas, a pesar de que todo el tiempo las critique y aduzca que estas valoraciones son subjetivas y variables. No obstante, son las únicas herramientas con que cuenta para evaluar los resultados de sus decisiones, las cuales provienen de los modelos económicos del racionalismo que permiten aplicar indicadores prescriptivos.

Por otra parte, un ejecutor debe tener claro que debe concebir por lo menos una decisión fundamental, para que luego, cuando tenga que tomar decisiones incrementales, no se vuelvan acciones sin un sentido práctico. Por esta razón el debate entre el modelo del racionalismo versus el modelo del incrementalista se vuelve superfluo, porque las herramientas del uno, terminan siendo utilizadas por el otro y viceversa.

Lo ideal para una buena toma de decisiones es que se aplique el modelo de exploración combinada, que, según Etzioni (1967, p. 274), consiste en iniciar procesos de decisión de políticas fundamentales y continuar con procesos incrementales de decisiones que preparen las decisiones fundamentales 0 las ejecuten, una vez adoptadas. Un ejemplo para ilustrar la estrategia es la estrategia de exploración combinada. Esta estrategia en la política de seguridad democrática desarrollada en Colombia permitió atacar las áreas que presentaban mayor problema, sin olvidar que se podrían presentar hechos violentos en áreas no previstas en la estrategia original que combinó la concentración de esfuerzos en algunas regiones consideradas estratégicas, con análisis exhaustivos de georeferenciación del conflicto, junto con una presencia temporal y selectiva de la fuerza pública, en las demás regiones del país.

En algunos periodos del gobierno se decidió intensificar la presencia civil y militar a través de redes de cooperantes en determinadas regiones, para así reflexionar mejor sobre las estrategias de guerra y buscar soluciones sostenibles a los problemas sociales de la comunidad; por esta razón se iniciaron nuevos programas tales como "El Plan retorno a las familias desplazadas" o "el Programa de reinserción y los procesos de justicia restaurativa", lo cuales han permitido visualizar los peligros y los riesgos propios que tiene la 
política de seguridad democrática, por lo que se ha aumentado la inversión en temas sociales y se han implementando programas tales como la "prevención al desplazamiento forzado, las Alertas Tempranas de la Defensoría del Pueblo".

Con respecto al aumento de inversión para restablecer los derechos de los desplazados, esta decisión se logró fruto del incremento marginal de los actores principales mediante una tutela fallada por la Corte Constitucional que permitió visualizar "un estado de cosas inconstitucional[es]", para más de dos millones de personas desplazadas, quienes tan sólo habían recibido una ayuda humanitaria por parte del Estado, y que se encontraban por fuera de cualquier programa de restablecimiento de las condiciones sociales y económicas. Esto generó el "Plan Integral de Atención a las Familias Desplazadas", que fue incorporado en el Plan de Desarrollo, aprobado en el segundo mandato del presidente Uribe, que comenzó en agosto de 2006. En síntesis, la exploración combinada de estrategias por parte del Gobierno Nacional ha proporcionado criterios o evidencias para las situaciones en las que es necesario enfatizar y destinar más recursos para la inversión social, para no desacreditar la política de "seguridad democrática".

Con este ejemplo se puede concluir que el debate entre racionalismo e incrementalismo es artificial, porque ambos se necesitan y un buen ejecutor debe saber que el incrementalismo reduce los aspectos poco realistas o demasiado teóricos del racionalismo, lo que limita los detalles en las decisiones fundamentales, mientras que el racionalismo permite que se superen los aspectos negativos del incrementalismo, tales como la posibilidad de explorar decisiones a largo plazo. En conclusión, el enfoque combinado permite unir lo empírico con lo teórico, lo que permite tomar decisiones más realistas y eficaces.

Una segunda aproximación al incrementalismo es la teoría del equilibrio puntuado. Ahora bien, cabe preguntarse si se logran solucionar las deficiencias del modelo del incrementalismo con la teoría reciente del equilibrio puntuado. La teoría del equilibrio puntuado (True, Jones \& Baumgarther, 1999, pp. 97-116) es un esquema de análisis flexible para explicar el proceso de estabilidad y de cambio en la elaboración de una política pública en un país, la que permite interpretar sus cambios, analizando sus ciclos y el papel que la incertidumbre y la contingencia han jugado en ella. Efectivamente, podría entrar a solucionar algunas deficiencias del incrementalismo que tiende a darle mayor énfasis a la coyuntura y a la contingencia, vista como una sumatoria de incrementos marginales de los actores principales, sin analizar su evolución histórica, la estabilidad de los grupos de interés y el tiempo que estos actores se han mantenido en el poder.

Según la teoría del equilibrio puntuado de Baumgarther y True, una característica de los sistemas políticos es que siempre se encuentran en equilibrio; esta estabilidad se mantiene por largos periodos, hasta que se presentan situaciones abruptas de cambio. Las razones para que haya prolongados periodos de estabilidad radican en la fuerza que ejercen las estructuras existentes de las políticas públicas institucionales y la definición de procesos temáticos por esas mismas instituciones.

Los cambios se producen porque no todos los intereses tienen la misma fuerza; las instituciones tienden a favorecer algunos de ellos sobre el resto, por un tiempo determinado y estas ventajas son mantenidas por largos periodos de tiempo, gracias a los arreglos institucionales que apoyan dichas políticas públicas.

El equilibrio se pierde no sólo por el realineamiento partidario, sino también por otras variables, como el papel de las elites o de las ideas; por ejemplo, se altera cuando se define un nuevo tema. En Colombia, el debate sobre la construcción del metro para Bogotá ha hecho que se pierda intem- 
pestivamente el punto de equilibrio del sistema político, en el ámbito nacional y local. Asimismo, se pierde cuando hay un cambio en las normas institucionales o jurisdiccionales, por ejemplo, la implementación del sistema acusatorio en la justicia penal colombiana generó cambios en los operadores judiciales. También se pierde cuando se registra la agitación de movimientos sociales y éstos son nuevos grupos de interés y los que afectan la fuerza de los monopolios e irrumpen en la escena política, lo que genera inestabilidad momentánea y luego cambios que se registran de manera rápida $y$, por lo general, dramática $o$ dolorosa; un ejemplo es las luchas indígenas por la tierra en contra de las petroleras o de los inversionistas en biocombustibles. Estos hechos llevan a la creación de un nuevo equilibrio, que permanecerá vigente por un tiempo prolongado.

Se concluye que, según esta teoría, los grupos de interés tienen su evolución, es decir, surgen, se consolidan y luego fracasan o colapsan. Por esta razón las políticas públicas se deben valorar si se estudian sus ciclos históricos y el papel que la incertidumbre y la contingencia han desempeñado en su implementación. Por lo tanto, la teoría del equilibrio puntuado podría llegar a superar las deficiencias del incrementalismo, puesto que cuando este enfoque observa el sistema político, con sus subsistemas en las organizaciones gubernamentales, su evolución y causas del cambio, logra explicar científicamente las pautas de la estabilidad y las del cambio, con una aproximación más científica y al mismo tiempo más realista.

En aras de abordar la tercera parte de este artículo, nos adentramos en el estudio de un cuarto modelo, el modelo del "cubo de basura" y el derivado de Kingdon. Al respecto surge la duda de si se trata de modelos racionales de adopción de decisiones $o$, por el contrario, si son modelos incrementalistas y si se puede llegar a la conclusión que estos modelos se podrían denominar "racionales comprensivos" por las siguientes razones.
En primer lugar, el modelo inicial fue desarrollado por Kingdon y luego perfeccionado por Zahariadis (1999, pp. 83-96). También se ha llamado "modelo de corrientes múltiples" o "ventana de oportunidades", porque pretende explicar los procesos de toma de decisiones en la definición de la agenda pública, a partir de la observación científica del comportamiento de las políticas públicas en sí mismas, las cuales atraviesan por largos periodos de estabilidad, y luego sufren de cambios radicales, que se atribuyen -según los resultados de las encuestas y las entrevistas practicadas por Kindong durante un periodo de cuatro- a la interacción de tres corrientes que intervienen de manera relativamente independiente en el proceso de toma de decisiones, en el cual tanto las estructuras institucionales que hacen las políticas como las estrategias individuales de la política de los empresarios juegan una función importante.

Las tres corrientes son: Ios problemas, las políticas y la política. En la primera corriente, "los problemas" están presentes en la información que tienen los diversos actores sobre el contexto social; éstos últimos son quienes proponen diversas soluciones, las cuales, en algunos casos, son radicales o desafiantes. Son reconocidos y actualizados a partir del estudio científico de la realidad. En el diseño de la agenda pública se pueden presentar choques entre las evaluaciones de la realidad con las que cuentan los estudiosos de la política y aquéllas que arrojan los nuevos datos científicos que pretenden causar reconocimiento entre la comunidad académica y, por ende, buscan la redefinición de los problemas y sus consecuencias.

Ejemplos de problemas actuales en Colombia y en el mundo, son, por una parte, los efectos del calentamiento global y su conexidad con el desarrollo del capitalismo y, por otra, los hechos terroristas transnacionales que cometen los grupos armados al margen de la ley y las actuaciones que se esperarían de los países miembros de la ONU, específicamente la lucha de Colombia 
contra las FARC y la posición que han asumido los países vecinos.

La segunda corriente: "las políticas" incluye una amplia variedad de ideas que se escuchan entre la gente y son la primera aproximación al problema, la mayoría de ellas son generadas por los funcionarios públicos y por los investigadores. En Colombia las facultades de Derecho tienen, en su mayoría, la línea de investigación de "Derecho Público y sociedad", lo que les permite hacer aportes importantes en las reformas que requiere la construcción del Estado social y democrático de derecho.

En la tercera corriente: "la política" se agrega la cualificación de los actores: los movimientos o grupos de presión económica o política, los cambios en el legislativo y la intervención de las agencias administrativas que encarnan la tecnocracia. En esta última se identifica el "humor nacional", las actividades de los grupos de presión y el volumen de negocios, los cuales pueden jugar un papel importante para la toma de decisiones. Si existe un número considerable de personas, con unas líneas de pensamiento comunes, esto se detecta en el humor nacional y se espera que el gobierno, los funcionarios y los políticos sean sensibles a este cambio.

En determinadas circunstancias, normalmente asociadas a las vertientes de la política o a las cuestiones externas al problema político, las relaciones entre actores se tornan candentes y es cuando se abre una ventana de oportunidades, que generalmente da lugar a la toma de decisiones, en las cuales los diseñadores de la agenda pública deben entrar a promulgar y a promover las nuevas políticas públicas, lo que genera, por lo general, cambios drásticos. Los cambios de personas dentro de los órganos de elección y las administraciones son la hipótesis que tienen un considerable impacto en la agenda política.

Entonces, las ventanas de oportunidad son momentos críticos, en los cuales los defensores de las nuevas políticas tienen la oportunidad de lograr captar la atención para sus problemas o para encontrar aprobar las nuevas soluciones políticas que vienen siendo aceptadas. Un ejemplo típico de estas ventanas que ofrecen oportunidades de cambio y la implementación de una política de choque es la elección de un gobernante de la capital de la República, que proviene del partido Polo Democrático, con una filosofía socialdemócrata y que fue elegido para dar continuidad a la política de "inclusión social" que inició el Alcalde saliente, al quedar demostrado que la inversión masiva en educación y en salud, mejora las condiciones de vida de la población y permite avances significativos hacia la modernidad y, por ende, hacia la paz. Si esta política de inclusión social se mantiene, se podría prever en dos años una disputa por la Presidencia de la República, en la que los grupos de presión generarían cambios radicales en el manejo del ejecutivo y en la política de seguridad democrática. Se anota que esta corriente política ha venido aprovechando estas ventanas de oportunidades, para demostrar que la paz también se puede conseguir incrementando la inversión social, con lo que podrían lograr el apoyo político mayoritario para este tipo de decisiones.

Un segundo ejemplo es el siguiente: la muerte del comandante Raúl Reyes con ocasión del enfrentamiento del Ejército colombiano y las FARC en territorio ecuatoriano está causando una crisis mundial. En este problema se evidencian las tres corrientes de las que habla el modelo de una "ventana de oportunidades", por cuanto están confluyendo las tres corrientes, lo que seguramente generará un cambio en las políticas públicas nacionales e internacionales, en torno a la persecución de los terroristas.

Con respecto a la primer corriente, "el problema", es claro que si Colombia está sola, no puede combatir a las FARC y si a esto se le suma la "ayuda" que los mandatarios de los países vecinos les están brindando a estos grupos insurgentes, la situación 
se agrava. Por lo tanto, se requiere la firma de unos protocolos en materia de Derecho Internacional Humanitario, que permitan la acción conjunta en las fronteras. En cuanto a la segunda corriente: las políticas, es evidente que desde el 11 de septiembre se generaron comentarios y acciones nacionales e internacionales específicas en torno a la persecución de los terroristas internacionales, las cuales constituyeron la primera aproximación al problema.

Con respecto a la tercera corriente: "la política contra el terrorismo", existe un "humor internacional", que se percibe tanto en los gobiernos de los países miembros de la ONU como en los órganos legislativos nacionales e internacionales, así como en los intereses precisos de los grupos económicos de presión, a los cuales les interesa que se apruebe el TLC o en su defecto que se avance en la globalización de la economía en América Latina, para lo cual se requiere la pacificación de Colombia; en consecuencia, aprobaría una nueva política pública contra el terrorismo internacional, que visualizaría un apoyo internacional a Colombia. Sin embargo, se está corriendo el riesgo de entrar en guerra con los países vecinos, por haber violado la soberanía, al incursionar el territorio ecuatoriano y dar de baja a los violentos. El problema no es fácil de resolver y Colombia hoy en día y más que nunca necesita apoyo de la diplomacia internacional, para no entrar en guerra y lograr plasmar una nueva política.

En síntesis, el modelo de Kingdon defiende la relativa independencia del problema, de la política y de las corrientes políticas durante el proceso de determinación de la agenda pública y explica el cambio de las políticas, por la exploración de las ventanas de oportunidad, por la misma política o por la acción de los políticos y empresarios opositores a la política pública actual.

Jhon Kingdon partió del modelo de comportamiento organizacional desarrollado por Cohen (Cohen;
March \& Olsen 1972, pp. 1-25) y elaboró su propio modelo en el que analizó el proceso de definición de las políticas en condiciones ambiguas, al constatar que las teorías tradicionales no arrojan resultados racionales para la toma de decisiones. En estos casos se vuelve crucial buscar una política alternativa, que dependa tanto de los problemas detectados como de los actores que quieran impulsar y proponer algunas soluciones.

Con los resultados de las entrevistas y encuestas, Kingdon defiende el concepto de ventana de oportunidades, para referirse a la oportunidad que tiene una determinada propuesta para ser considerada o adoptada por una administración. Las ventanas de oportunidades se abren cuando confluyen tres factores: un problema reconocido, la existencia de una posible solución que ya ha sido probada por una comunidad de expertos políticos, apoyada por académicos lo que permite que se produzca el cambio, al ser el momento adecuado para esa nueva orientación política.

Zahariadis amplía el modelo de Kingdon y ofrece respuesta a tres cuestiones esenciales en la definición de la agenda pública:

- ¿A qué deben prestar atención los "diseñadores de la agenda pública" para focalizar algunos problemas y algunas decisiones?

- ¿Cómo están determinadas y moldeadas las relaciones entre actores?

- ¿Cómo y cuándo se deben buscar las soluciones?

Una de las primeras críticas consiste en que Kingdon no trata a los actores políticos como grupos capaces de ejercer una acción estratégica. Los modelos desarrollados más adelante, como la teoría del equilibrio puntuado (True et al., 1999, pp.97-116), explican que además de la lógica de la estabilidad y de los incrementos, ocasionalmente se producen en gran escala salidas del pasado. "Statu quo" en lugar de crisis, en los que 
caracteriza la mayoría de ámbitos políticos; esta teoría trata de explicar el statu quo y las crisis en un modelo y hace énfasis en la definición y en el establecimiento de los programas.

Asimismo, en esta teoría se explica que los actores políticos, por un lado, tratan de controlar las imágenes de los problemas a través del uso de la retórica, de los símbolos y de la ciencia política. Por otra parte, tratan de alterar la lista de participantes que están involucrados en la búsqueda de la solución más favorable. Cuestionar las políticas entre actores fundamentales crea las condiciones para dramáticos retrocesos.

El modelo de "la agenda de política dinámica y subsistemas de la política" (Baumgartner \& Jones, 1991, pp. 1044 1074) avanza más en la explicación del diseño de la agenda pública, al dar cuenta de la forma como se crean y se destruyen los subsistemas de la política. Se explica que lo normal es que en un sistema político pluralista se creen subsistemas que son altamente favorables a una política determinada, pero, al mismo tiempo, existen otros actores que más adelante servirán de fuerza para el cambio. El proceso de diseño de la agenda pública atraviesa por la interacción de las creencias y de los valores sobre una política en particular, asimismo, requiere de una política de imagen que permita la estabilidad de la política por largos periodos de tiempo. Este modelo avanza en la explicación de las relaciones entre los diferentes actores o corrientes.

Se infiere que los modelos antes vistos sirven para explicar el diseño de la agenda pública, es decir, son complementarios y convergen en que en todos los sistemas pluralistas y ampliamente democráticos se evidencian periodos de extrema estabilidad y periodos de crisis y que los diferentes actores juegan un papel primordial en el diseño de las nuevas políticas públicas.
Por último, se analizarán y se sintetizarán los modelos más recientes de enfoque de políticas públicas: el modelo propuesto por Scharpf y el modelo de Sabatier. El modelo de coaliciones promotoras desarrollado por Paul Sabatier (1988, pp. 130-ss) considera que las políticas públicas son el resultado de la competición y de la lucha entre coaliciones promotoras de las diferentes opciones de política pública dentro de los subsistemas' ${ }^{1}$.

En lo interno, este modelo se centra en estudiar las coaliciones y las creencias básicas que comparten y la política orientada hacia el aprendizaje; en lo externo, el modelo se centra en buscar la explicación de los eventos del subsistema que inciden en el cambio radical de la política pública. El núcleo profundo de un sistema de creencias básicas incluye un criterio ontológico y un sistema normativo. Estas creencias se refieren, por ejemplo, a la valoración relativa de la libertad individual, frente a la igualdad social, o a la percepción de la relación entre el ser humano y la naturaleza. Estas creencias tienen una estructura tripartita: la percepción sobre la gravedad del problema; la percepción sobre sus principales

1 Un subsistema de políticas públicas es una unidad de análisis que utiliza Sabatier para comprender el cambio en las políticas; está integrado por actores de organizaciones públicas y privadas que están activamente implicados con un problema de política pública. En un subsistema político pueden existir varias coaliciones. Por otra parte, por coaliciones promotoras se entiende toda agrupación de actores en un subsistema de políticas públicas, que comparte un sistema de creencias y de información dado, en términos de valores, asunciones causales, percepciones sobre el problema público y posibles soluciones. Los actores se pueden agrupar en tres coaliciones: a) los líderes de cada grupo de interés; b) la agencia de funcionarios públicos, en su mayoría tecnócratas, junto con los investigadores de ciencias sociales aplicadas, académicos y periodistas; y c) los políticos propiamente dichos o congresistas. Estas coaliciones están integradas; sin embargo, las partes en una coalición diagnostican los problemas y, al mismo tiempo, debaten las alternativas políticas. Todos estos actores, en el marco de la coalición, comparten un conjunto de creencias y muestran un grado significativo de comportamiento estratégico coordinado, lo que les permita alcanzar sus objetivos e imponer sus propuestas de políticas. Los grupos son identificados como centrales, para la explicación de la dinámica de la acción política realizada sobre las políticas públicas, por ejemplo, el grupo de los científicos sociales o académicos hace parte de la segunda coalición y tiene como característica que sus miembros están motivados por las preocupaciones políticas, por esta razón logran incidir en el diseño de las políticas públicas. 
causas y la percepción acerca de los mecanismos institucionales para hacer frente al problema.

Con respecto al manejo de la incertidumbre, la información y las creencias, Sabatier considera que el uso y la acumulación de la información es un elemento esencial del cambio de las políticas. Las creencias de los actores sirven como filtros perceptivos en la recepción de información, no obstante, la información puede cambiar las creencias.

Por lo general, las coaliciones son resistentes al cambio radical y logran cierta inercia o estabilidad en el tiempo; sin embargo, los cambios se logran luego del aprendizaje a través de los sistemas de creencias de las diferentes coaliciones. Como cada coalición trata de aprender más acerca de las posibilidades de Ilegar a lograr los objetivos de la política, la coalición de investigadores sociales es la que más contribuye al aprendizaje de las otras coaliciones.

Según esta teoría, las redes de individuos son las que siguen siendo los principales agentes del cambio. El modelo le da a las "ideas", un papel fundamental, al indicar que la información científica y los procesos de aprendizaje permiten mejorar la formulación de las políticas. El modelo también plantea que las perturbaciones externas a los subsistemas de políticas públicas son necesarias para el cambio; ejemplo de ellas son los cambios en las condiciones socioeconómicas, los cambios en la opinión pública, los cambios en el sistema que rige las coaliciones; por esta razón, el papel de la información, en general, es fundamental para comprender la dinámica del cambio de las políticas. Los actores procesan y filtran información para apoyar un sistema de creencias dado o para cambiarlo.

La dinámica sobre la toma de decisiones y del cambio en las políticas públicas se concibe en función de:
- La interacción de coaliciones promotoras adversarias dentro de un subsistema de políticas;

- Los cambios externos en el subsistema, por ejemplo, las condiciones socioeconómicas, y

- Los efectos de parámetros relativamente estables del sistema, por ejemplo, las disposiciones constitucionales o la estructura social básica.

Entre las deficiencias que se le han detectado al modelo se encuentra que asume la formación de grupos y de colaciones, pero no las explica; tampoco toma en cuenta el comportamiento estratégico y oportunista de los grupos ni le otorga una atención sustancial a estas acciones colectivas. El modelo de Fritz Scharpf (1997), denominado institucionalismo centrado en actores, ofrece llenar los vacíos del modelo de Sabatier, al introducir dos elementos más: la constelación de actores y los modos de interacción entre actores e institucionalidad. Scharpf destaca la incidencia que tienen las instituciones por un lado, sobre las percepciones, las preferencias y las capacidades de actores individuales o colectivos y, por otro, las formas en que estos actores interactúan.

Las instituciones son la principal fuente de información de los actores y el principal factor que influye sobre las decisiones, en el sentido que permiten reducir o aumentar algunos incentivos para escoger determinadas estrategias de acción. Sin embargo, los actores continúan con un amplio margen de discrecionalidad para elegir sus tácticas y obtener los resultados preconcebidos.

En este modelo cada actor está institucionalmente constituido, pues emerge basado en reglas preexistentes, reglas que aseguran su permanencia y actuación. Por ejemplo, estas reglas definen los recursos disponibles, la esfera de sus actividades legítimas, el alcance de sus representantes, inclusive, sus principios rectores.

Por su parte, las instituciones son las que delimitan cómo se debe evaluar el actor implicado en los resul- 
tados de tales elecciones y determinan las preferencias del actor con respecto a las alternativas posibles. Así como las instituciones permiten la estructuración de cada actor, también constituyen una fuente de información para predicciones mutuas con respecto a cómo actuarán o no actuarán los otros actores implicados en determinada área política.

Al igual que Sabatier, en el modelo de Sharif, los actores están caracterizados por la orientación de sus acciones, es decir, por determinadas creencias que pueden ser relativamente invariables o pueden cambiar mediante el aprendizaje y la persuasión. El actor se cuestiona sobre el problema por resolver, el estado de la temática, las causas, la valoración con respecto a las políticas posibles y los resultados probables. Sin embargo, para ello, se parte de actores individuales y colectivos, que forman parte del proceso de elaboración de la política pública concreta y cuyas decisiones determinan los contenidos de la política.

La diferencia con Sabatier consiste en que en este modelo lo que prevalece no es el actor individual, sino que se introduce el elemento de "una constelación de actores implicados en esa interacción política", por lo que se requiere esquematizar el problema en función a esa constelación de actores que están tomando parte de esa definición. Por ejemplo, si lo que se está analizando es la política de reparación a las víctimas en Colombia, la constelación de actores incluye el gobierno y los representantes de las Organizaciones Internacionales y ONG nacionales defensoras de Derechos Humanos. En función de las estrategias que decida cada uno de los actores, se producen diferentes resultados en términos de política de justicia y paz, que serán valorados de manera distinta por cada uno de ellos.

Un tercer elemento que introduce este autor son "los modos de interacción", el cual se refiere a las formas en que unos actores se conducen con respecto a los otros, los cuales están en, gran medida, condicionados por el contexto institucional en el que se desenvuelven. Cada modo de interacción está regulado por un sistema de pautas institucionales para el uso de ese modo en cuestión y está influenciado por un sistema institucional más amplio, que también influyen en las decisiones. En el caso antes señalado de la Política de Justicia y Paz en Colombia, serían:

- Escenarios de grupos de víctimas con mínima organización social;

- Sistemas de justicia restaurativa, que conviven con sistemas de justicia interamericana;

- Asociaciones de desplazados, grupos de familiares de las víctimas, grupos de académicos y de juristas, $y$

- El sistema judicial tradicional colombiano.

El hecho de haber introducido estos dos elementos nuevos en el modelo de Sharpf, permite una mayor comprensión de los resultados de determinada interacción política en un país y de cómo se legitima el poder.

\section{CONCLUSIÓN}

Ya analizados los modelos existentes para la elaboración de las políticas públicas, se concluye que lo ideal para que los gobernantes de América Latina y de Colombia logren tomar buenas decisiones es que empiecen a aplicar modelos de manera combinada, con los cuales intercambien decisiones fundamentales, junto con decisiones incrementales; esto permitiá enfatizar en los temas que vayan surgiendo como prioritarios y destinar más recursos de los que lo exijan los diferentes actores.

La teoría del equilibrio puntuado enseña que los sistemas políticos logran equilibrios por largos periodos de tiempo, lo que radica en la fuerza que ejercen las estructuras existentes, que, a su vez, logran arreglos institucionales para apoyar determinadas políticas públicas; sin embargo, 
estos equilibrios no son eternos. Los cambios se producen, porque no todos los intereses conservan la misma fuerza. Cuando se define un nuevo tema, por ejemplo, la seguridad democrática o el metro para Bogotá, se pierde intempestivamente el punto de equilibrio del sistema político.

También se pierde cuando hay cambio en las normas institucionales, lo que permite inferir que en Colombia se presentarán nuevas corrientes, con ocasión de la implementación del nuevo sistema de administración de justicia contencioso administrativa o la nueva reforma tributaria para los entes territoriales, que hoy en día se están gestando entre los académicos para ser presentadas como proyecto de ley, ante el Congreso.

Asimismo, el equilibrio se pierde con la agitación de los movimientos sociales y son éstos nuevos grupos de interés, los que afectan la fuerza de los monopolios e irrumpen en la escena política, lo que generando inestabilidad momentánea y luego cambios que se registran de manera rápida y, por lo general, dramática o dolorosa. Ejemplos de estos cambios son la lucha de las negritudes y de los indígenas por la titulación de sus tierras; la lucha de las familias desplazadas y las víctimas de la violencia por lograr su reparación, o la de las mujeres por lograr la igualdad real en el ámbito laboral. Estos movimientos sociales están afectando el equilibrio político y están generando cambio en las políticas públicas, en lo que a ellos conciernen. Por estas razones, el análisis de las políticas públicas en Colombia se debe hacer observando los ciclos históricos, dejando que la incertidumbre y la contingencia cumplan también su rol, para poder desentrañar cómo ha sido su verdadera implementación y cuál será su futuro.

Los movimientos sociales y los académicos tendrán que ir perfeccionando sus herramientas para captar la atención de los demás actores políticos y económicos, y así poder aprovechar al máximo las ventanas de oportunidad que se les presenten; en otras palabras, estar atentos a defender en diferentes escenarios políticos y sociales las soluciones que ellos mismos propongan. Por último, gracias al modelo de la agenda de política dinámica y subsistemas de la política, nuestros gobernantes están entendiendo que existen unos subsistemas de la política, los cuales se crean y se destruyen, que pueden llegar a ser favorables o desfavorables para la implementación de determinada agenda, por esta razón los diversos actores deberán interactuar con las creencias y con los valores para poder llevar a cabo su cometido de implementar la política deseada, para lo cual deberán esforzarse por entender cómo se relacionan e interactúan las diferentes corrientes.

Para concluir, todos los modelos vistos son complementarios y sirven a los actores de la realidad colombiana, por cuanto se desarrollan en esquemas pluralistas como el nuestro, con la tarea de ser ampliamente democráticos. Los actores deben aprenden a jugar y a actuar en colectivo, con estos subsistemas de poder, de tal manera que logren periodos de estabilidad, sin desconocer que los periodos de crisis son muy importantes para avanzar en la construcción de un Estado Social y Democrático de Derecho, como el que añoramos los colombianos.

\section{REFERENCIAS}

Baumgarther, F.R. \& Jones, B.D. (1991). Agenda Dynamics and Policy Subsystems. Journal of Politics.

Bukowski, J. (2007). Spanish Water Policy and the National Hydrological Plan: An Advocacy Coalition Approach to Policy Change. South European Society and Politics, 12, (1).

Cohen, M.D.; March, J.G. \& Olsen, J.P. (1972). A Garbage Can Model of Organizational Choice. Administrative Science Quarterly, 17 (1). 
Etzioni, A. (1967). La exploración combinada: un tercer enfoque en la toma de decisiones. Revista de Administración Pública, 27.

Meijerink, S. (6 $6^{\text {th }}$ December, 2005). Understanding Policy Stability and Change. The Interplay of Advocacy Coalitions and Epistemic Communities, Windows of Opportunity and Dutch Coastal Flooding Policy 1945-2003. Journal of European Public Policy, 12.

Scharpf, F. (1997). Games Real Actors Play. ActorCentered Institutionalism in Policy Research. Boulder: Westview.
Sabatier, P.A. (1988). An Advocacy Coalition Framework of Policy Change and the Role of PolicyOriented Learning Therein. Policy Sciences, 2

True, J.L.; Bryan, D.J. \& Baumgartner, F.R. (1999). Punctuated-Equilibrium Theory. Explaining Stability and Change in American Policymaking. In Paul A. Sabatier (editor). Theories of the Policy Process. Boulder: Westwiew Press.

True, J.L.; Bryan, D.J. \& Baumgarther, F.R. (1999). Punctuated Equilibriun Theory. Editor Paul Sabatier.

Zahariadis, Nikolaos. (1999). Ambiguity, Time, and Multiple Streams. In Paul A. Sabatier (ed.). Theories of the Policy Process. Boulder: Westview Press. 